\title{
Experimental determination of isotope enrichment factors - bias from mass removal by repetitive sampling
}

Buchner, Daniel; Jin, Biao; Ebert, Karin; Rolle, Massimo; Elsner, Martin; Haderlein, Stefan B.

\section{Published in:}

Environmental Science and Technology

Link to article, DOI:

10.1021/acs.est.6b03689

Publication date:

2017

Document Version

Publisher's PDF, also known as Version of record

Link back to DTU Orbit

Citation (APA):

Buchner, D., Jin, B., Ebert, K., Rolle, M., Elsner, M., \& Haderlein, S. B. (2017). Experimental determination of isotope enrichment factors - bias from mass removal by repetitive sampling. Environmental Science and Technology, 51(3), 1527-1536. https://doi.org/10.1021/acs.est.6b03689

\section{General rights}

Copyright and moral rights for the publications made accessible in the public portal are retained by the authors and/or other copyright owners and it is a condition of accessing publications that users recognise and abide by the legal requirements associated with these rights.

- Users may download and print one copy of any publication from the public portal for the purpose of private study or research.

- You may not further distribute the material or use it for any profit-making activity or commercial gain

- You may freely distribute the URL identifying the publication in the public portal 


\title{
Experimental Determination of Isotope Enrichment Factors - Bias from Mass Removal by Repetitive Sampling
}

\author{
Daniel Buchner, ${ }^{\dagger}$ Biao Jin, ${ }^{\dagger, \perp}$ Karin Ebert, ${ }^{\dagger, \S}$ Massimo Rolle, ${ }^{\dagger, \perp}$ Martin Elsner, $\|$ \\ and Stefan B. Haderlein $* \dagger$
${ }^{\dagger}$ Department of Geosciences, Center for Applied Geosciences, University of Tübingen, Hölderlinstraße 12, D-72074 Tübingen, Germany
"Institute of Groundwater Ecology, Helmholtz Zentrum München, Ingolstädter Landstraße 1, D-85764 Neuherberg, Germany
${ }^{\perp}$ Department of Environmental Engineering, Technical University of Denmark, Miljøvej Building 113, DK-2800 Kgs. Lyngby, Denmark

Supporting Information

\begin{abstract}
Application of compound-specific stable isotope approaches often involves comparisons of isotope enrichment factors $(\varepsilon)$. Experimental determination of $\varepsilon$-values is based on the Rayleigh equation, which relates the change in measured isotope ratios to the decreasing substrate fractions and is valid for closed systems. Even in well-controlled batch experiments, however, this requirement is not necessarily fulfilled, since repetitive sampling can remove a significant fraction of the analyte. For volatile compounds the need for appropriate corrections is most evident, and various methods have been proposed to account for mass removal and for volatilization into the headspace. In this study we use both synthetic and experimental data to demonstrate that the determination of

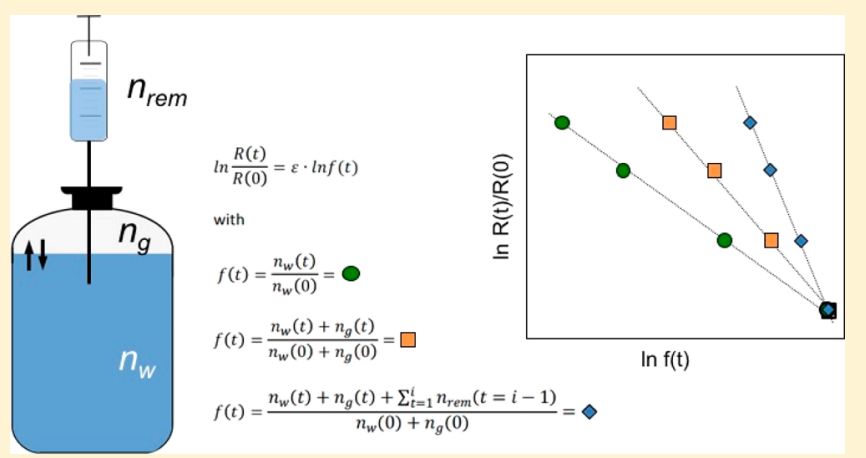
$\varepsilon$-values according to current correction methods is prone to considerable systematic errors even in well-designed experimental setups. Application of inappropriate methods may lead to incorrect and inconsistent $\varepsilon$-values entailing misinterpretations regarding the processes underlying isotope fractionation. In fact, our results suggest that artifacts arising from inappropriate data evaluation might contribute to the variability of published $\varepsilon$-values. In response, we present novel, adequate methods to eliminate systematic errors in data evaluation. A model-based sensitivity analysis serves to reveal the most crucial experimental parameters and can be used for future experimental design to obtain correct $\varepsilon$-values allowing mechanistic interpretations.
\end{abstract}

\section{INTRODUCTION}

Compound specific isotope analysis (CSIA) is widely applied in various fields of environmental sciences (i) as a forensic tool for contaminant source identification, ${ }^{1,2}$ (ii) to detect and quantify in situ (bio)-degradation, ${ }^{3-5}$ and (iii) for process characterization including identification of transformation mechanisms in (bio)-degradation studies. ${ }^{6-11}$ The latter two applications involve isotope enrichment factors $(\varepsilon)$, which relate quantitatively measured isotope ratios to substrate fractions of an analyte. $\varepsilon$-values are usually determined using laboratory batch experiments and may be lumped parameters, not only reflecting the underlying kinetic isotope effect of a reaction but also physical constraints or experimental artifacts. Thus, reported $\varepsilon$-values for a given process may span a wide range; for instance, the published $\varepsilon_{\text {carbon-values for reductive dehalogenation of }}$ trichloroethene (TCE) range from $-2.5 \%$ to $-19.6 \% o^{7,12-14}$ Numerous experimental studies tried to unravel the processes and mechanisms that determine the extent of isotope fractionation for a given reaction, e.g., by investigating the transport of the substrate across the cell wall, ${ }^{7,8}$ the efficacy of the involved enzymes, ${ }^{15}$ the cell density, ${ }^{9,16}$ or the nutrient supply. ${ }^{13,17}$

For closed systems, isotope enrichment factors can be obtained using a simplified form of the Rayleigh distillation equation ${ }^{18,19}$ where $\varepsilon$ is derived from the slope of the linear correlation between the natural logarithm of the measured substrate fraction, $\ln f_{\text {substrate }}(t)$, and the measured isotope ratios, $\ln$ $(R(t) / R(0))$. While it is obvious that field sites often cannot be treated as closed systems and require modified forms of the Rayleigh equation, ${ }^{4,20}$ for laboratory batch reactors the applicability of the linearized form of the Rayleigh equation seems to be straightforward as such systems appear to fulfill all requirements of closed systems. These conditions are strictly met in experiments with numerous parallel replicates which are sacrificed sequentially for sampling, ${ }^{7,8,15}$ however, at the expense

Received: July 22, 2016

Revised: October 31, 2016

Accepted: November 22, 2016

Published: November 22, 2016 

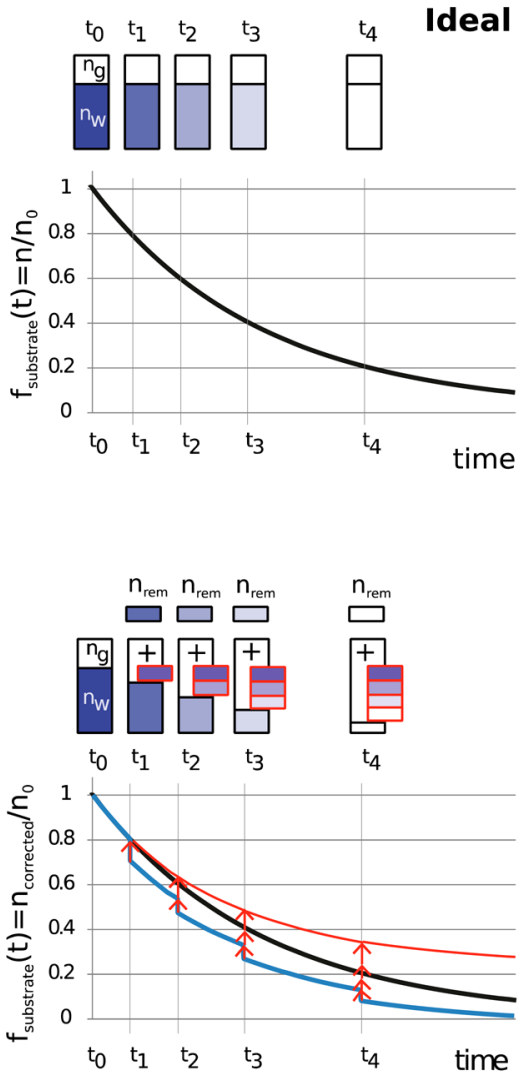

Inadequate

Correction

(method I-III)

time

Ideal Case

Figure 1. Schematic illustration of the time course of the substrate fraction, $f$, during biodegradation in a repetitively sampled batch reactor considering an ideal case in which neither compound's mass nor water volume are removed from the system and a realistic case which requires correction schemes. The figure illustrates substrate removal due to sampling by lower water levels ("slices" withdrawn) and removal due to biodegradation by concentration changes (color shades). For example, method III (Henry + CSR) adequately corrects for mass removal by sampling $\left(n_{\mathrm{rem}}\right)$ but ignores that the removed mass cannot undergo further biodegradation as it would occur in an undisturbed reactor. When all amounts are summed up, this scheme therefore overestimates the remaining substance fraction $f_{\text {substrate }}(t)$ (red curve).

of increased uncertainty and variability due to different dynamics in such parallel assays.

Alternatively, several samples can be withdrawn from the same batch reactor over time to monitor isotope ratios at different values of $f_{\text {substrate }}(t)$. Repetitive withdrawal of water and/or gas samples from batch reactors violates the closed system requirement and calls for adequate consideration. Unfortunately, $\varepsilon$-values reported in the literature often lack a clear description whether corrections of measured concentration data were applied. Repetitive sampling from a batch reactor not only leads to mass removal of the target compound(s) from the system but also, if water samples are withdrawn, changes the phase ratio by decreasing the water phase and increasing the headspace volume. Progressive change of the phase ratio, however, needs to be taken into account for volatile organic contaminants such as chlorinated ethenes. As either gas or water volume is sampled, the measured data represent the concentration in only one of these phases, and a mass balance on the system is required using Henry's law and the gas/water ratio to account for the entire amount of substrate or products. $^{21,22}$ Additional corrections were suggested to account for the cumulative amount of substrate removed by repetitive sampling (CSR). ${ }^{13,14,23}$

The present study systematically investigates how such correction methods affect $\varepsilon$-values determined in batch experiments with repetitive sample removal. Using both experimental case studies as well as a synthetic data set calculated for typical experimental conditions, we evaluate the performance of various correction methods, identify pitfalls, and propose novel methods to eliminate systematic errors in data evaluation. A model-based sensitivity analysis was performed to identify which experimental parameters require the most stringent control and should be used to optimize the experimental design in order to obtain correct isotope enrichment factors that are suitable for mechanistic interpretation and thus indicative of the underlying isotope fractionation processes.

Methods to Determine Isotope Enrichment Factors. We describe current and newly proposed methods to account for mass removal by repetitive sampling in batch reactors considering volatile organic compounds. Based on the substrate fraction and stable isotope data the isotope enrichment factor $(\varepsilon)$ can be calculated using a simplified Rayleigh distillation equation $^{18,19}$ 


$$
\ln \frac{R(t)}{R(0)} \approx \varepsilon \cdot \ln f_{\text {substrate }}(t)
$$

where $R(t)$ and $R(0)$ are the stable isotope ratios of the substrate at a time $t$ and the initial time, and $f_{\text {substrate }}(t)$ denotes the remaining substrate fraction (often approximated by the substrate concentration according to $c(t) / c(0))$ at a time $t$. $\varepsilon$-values are derived by linear regression of the data without forcing the regression through the origin as suggested by Scott et al. ${ }^{24}$

The substrate fractions, $t$ and the initial time, and $f_{\text {substrate }}(t)$, at different time points come from measured concentration data of water samples subject to corrections accounting for volatilization into the increasing headspace and repetitive removal of water samples from the batch reactors. Figure 1 illustrates the effect of sampling and the challenges to adequately consider the effects of mass removal and volatilization.

Below, we outline current (I-III) and newly proposed methods (IV and V) to calculate the substrate fraction:

(I) The substrate fraction in water phase (water) without further corrections is calculated as follows

$$
f_{\text {water }}(t)=\frac{c_{\mathrm{w}}(t) \cdot V_{\mathrm{w}}(t)}{c_{\mathrm{w}}(0) \cdot V_{\mathrm{w}}(0)}=\frac{n_{\mathrm{w}}(t)}{n_{\mathrm{w}}(0)}
$$

where $n_{\mathrm{w}}(t)$ and $n_{\mathrm{w}}(0)$ correspond to the amount (moles) of the substrate in the water phase at times $t$ and $t=0$, respectively. Measured aqueous analyte concentrations, $c_{\mathrm{w}}(t)$, are multiplied by the water volume present at the respective time, $V_{\mathrm{w}}(t)$, to obtain $n_{\mathrm{w}}(t)$ thus accounting for the changes of the water volume.

(II) The correction for gas-water-partitioning (Henry) considers the total amount of substrate in the system by accounting for both the water phase and the gas phase of the batch reactor. The substrate fraction, $f_{\text {Henry }}(t)$, is calculated as

$$
f_{\text {Henry }}(t)=\frac{c_{\mathrm{w}}(t) \cdot V_{\mathrm{w}}(t)+c_{\mathrm{g}}(t) \cdot V_{\mathrm{g}}(t)}{c_{\mathrm{w}}(0) \cdot V_{\mathrm{w}}(0)+c_{\mathrm{g}}(0) \cdot V_{\mathrm{g}}(0)}=\frac{n_{\mathrm{w}}(t)+n_{\mathrm{g}}(t)}{n_{\mathrm{w}}(0)+n_{\mathrm{g}}(0)}
$$

where $n_{\mathrm{g}}(t)$ and $n_{g}(0)$ correspond to the amount of substrate in the gas phase at time $t$ and the initial time. The substrate concentration in the gas phase, $c_{\mathrm{g}}(t)$, is calculated from $c_{\mathrm{w}}(t)$ by applying Henry's law. $K_{\mathrm{H}}$ is the dimensionless Henry-constant $\left(K_{\mathrm{H}}=c_{\mathrm{w}} / c_{\mathrm{g}}\right)$ of the analyte at the appropriate temperature, calculated for $21^{\circ} \mathrm{C}$ according to Sander ${ }^{25}$ and references therein. $V_{\mathrm{g}}(t)$ is the volume of the gas phase in the batch reactor at time $t$.

(III) The correction for gas-water partitioning and cumulative sample removal (Henry+CSR) is an extension of the Henry-correction taking into account the amount of substrate removed by repetitive sampling of the reactor. The substrate fraction, $f_{\text {Henry }+\mathrm{CSR}}(t)$, is calculated according to

$$
\begin{aligned}
f_{\text {Henry }+ \text { CSR }}(t) & =\frac{c_{\mathrm{w}}(t) \cdot V_{\mathrm{w}}(t)+c_{\mathrm{g}}(t) \cdot V_{\mathrm{g}}(t)+\sum_{t=1}^{i}\left[c_{\mathrm{w}}(t=i-1) \cdot V_{\text {rem }}(t=i-1)\right]}{c_{\mathrm{w}}(0) \cdot V_{\mathrm{w}}(0)+c_{\mathrm{g}}(0) \cdot V_{\mathrm{g}}(0)} \\
& =\frac{n_{\mathrm{w}}(t)+n_{\mathrm{g}}(t)+\sum_{t=1}^{i} n_{\text {rem }}(t=i-1)}{n_{\mathrm{w}}(0)+n_{\mathrm{g}}(0)}
\end{aligned}
$$

where $n_{\text {rem }}(t)$ corresponds to the amount of the substrate removed by sampling at a given time $t$ and is calculated by multiplying the concentration in the water phase $\left(c_{w}(t)\right)$ with the removed volume $\left(V_{\text {rem }}(t)\right)$ at time $t$. The summation term denotes the cumulative amount of substrate removed from the system by sampling except for the last sampling. Even though this method adequately calculates the mass of substrate that is removed by sampling, it considers this mass as part of the substrate remaining fraction, as illustrated by the color shade in Figure 1. Even so method III accounts for the removed mass as it treats this mass as being part of the system that is subject to further (bio)-transformation. Consequently, the method overestimates the fraction of substrate remaining $f_{\text {substrate }}(t)$.

(IV) The stepwise correction (SW) method also accounts for gas-water partitioning and sample removal. Contrary to method III (Henry+CSR) this method applies an iterative correction scheme and avoids systematic over- or underestimation of substrate fractions which is conceptually inherent to methods I-III.

First, for two consecutive sampling times individual substrate fractions, $f_{(t-1) \rightarrow t}$, are calculated. By comparing the amounts of substrate present in the system at times $t$ and at time $(t-1)$ after sample withdrawal, this fraction represents exclusively changes due to (bio)-degradation:

$$
\begin{aligned}
& f_{(t-1) \rightarrow t}=\frac{n_{\mathrm{w}}(t)+n_{\mathrm{g}}(t)}{n_{\mathrm{w}}(t-1)+n_{\mathrm{g}}(t-1)-n_{\mathrm{rem}}(t-1)} \\
& \quad=\frac{c_{\mathrm{w}}(t) \cdot V_{\mathrm{w}}(t)+c_{\mathrm{g}}(t) \cdot V_{\mathrm{g}}(t)}{c_{\mathrm{w}}(t-1) \cdot V_{\mathrm{w}}(t-1)+c_{\mathrm{g}}(t-1) \cdot V_{\mathrm{g}}(t-1)-c_{\mathrm{w}}(t-1) \cdot V_{\mathrm{rem}}(t-1)}
\end{aligned}
$$

Using the dimensionless Henry's constant $\left(K_{\mathrm{H}}\right)$ eq 5 can be rearranged to

$$
\begin{aligned}
f_{(t-1) \rightarrow t} & =\frac{c_{\mathrm{w}}(t) \cdot V_{\mathrm{w}}(t)+\frac{c_{\mathrm{w}}(t) \cdot V_{\mathrm{g}}(t)}{K_{\mathrm{H}}}}{c_{\mathrm{w}}(t-1) \cdot V_{\mathrm{w}}(t-1)+\frac{c_{\mathrm{w}}(t-1) \cdot V_{\mathrm{g}}(t-1)}{K_{\mathrm{H}}}-c_{\mathrm{w}}(t-1) \cdot V_{\mathrm{rem}}(t-1)} \\
= & \frac{c_{\mathrm{w}}(t) \cdot\left[V_{\mathrm{w}}(t)+\frac{V_{\mathrm{g}}(t)}{K_{\mathrm{H}}}\right]}{c_{\mathrm{w}}(t-1) \cdot\left[V_{\mathrm{w}}(t-1)+\frac{V_{\mathrm{g}}(t-1)}{K_{\mathrm{H}}}-V_{\mathrm{rem}}(t-1)\right]}
\end{aligned}
$$

where $c_{\mathrm{w}}(t)$ and $c_{\mathrm{w}}(t-1)$ or $V_{\text {rem }}(t)$ and $V_{\text {rem }}(t-1)$ denote the measured concentrations or sample volumes taken at time $t$ and the preceding time point $t-1$.

Second, the overall substrate fraction at time $t$ is calculated considering $f(0)=1$ (i.e., $100 \%$ substrate fraction) and the results of eq 6 for all preceding time steps:

$$
f_{\mathrm{SW}}(t)=f(0) \cdot f_{t 0 \rightarrow t 1} \cdot f_{t 1 \rightarrow t 2} \cdot \ldots \cdot f_{t(n-1) \rightarrow t n}
$$

As an example, for two sampling steps this method leads to the following expression:

$$
\begin{aligned}
f_{\mathrm{SW}}(2) & =f(0) \cdot f_{t 0 \rightarrow t 1} \cdot f_{t 1 \rightarrow t 2} \\
& =1 \cdot \frac{c_{\mathrm{w}}(1) \cdot\left[\frac{V_{\mathrm{g}}(1)}{K_{\mathrm{H}}}+V_{\mathrm{w}}(1)\right]}{c_{\mathrm{w}}(0) \cdot\left[V_{\mathrm{w}}(0)+\frac{V_{\mathrm{g}}(0)}{K_{\mathrm{H}}}-V_{\mathrm{rem}}(0)\right]} \cdot \frac{c_{\mathrm{w}}(2) \cdot\left[\frac{\mathrm{V}_{\mathrm{g}}(2)}{K_{\mathrm{H}}}+V_{\mathrm{w}}(2)\right]}{c_{\mathrm{w}}(1) \cdot\left[V_{\mathrm{w}}(1)+\frac{V_{\mathrm{g}}(1)}{K_{\mathrm{H}}}-V_{\mathrm{rem}}(1)\right]}
\end{aligned}
$$

As can be seen, the terms representing the substrate concentration of the intermediate time point $t=1$ cancel out, and eq 8 simplifies to

$$
f_{\text {SW }}(2)=1 \cdot \frac{\left[\frac{V_{g}(1)}{K_{H}}+V_{w}(1)\right]}{c_{w}(0) \cdot\left[V_{w}(0)+\frac{V_{g}(0)}{K_{H}}-V_{\text {rem }}(0)\right]} \cdot \frac{c_{w}(2) \cdot\left[\frac{V_{g}(2)}{K_{H}}+V_{w}(2)\right]}{\left[V_{w}(1)+\frac{V_{g}(1)}{K_{H}}-V_{\text {rem }}(1)\right]}
$$

Note that this stepwise correction method requires the phase volume ratios for each time step but concentration measurements only for the initial and the final time step. 
Thereby the propagation of measurement errors (e.g., detector drift) from intermediate sampling times are minimized.

(V) The mass balance correction (MB) method determines the substrate fractionation, $f_{\mathrm{MB}}(t)$, from a mass balance of reactant and products for each time point, i.e., by dividing the amount of the substrate at time $t$ by the sum of the amounts of substrate and all degradation products at that time:

$$
\begin{aligned}
& f_{\mathrm{MB}}(t) \\
& =\frac{c_{\mathrm{w}, \text { substrate }}(t) \cdot V_{\mathrm{w}}(t)+c_{\mathrm{g}, \text { substrate }}(t) \cdot V_{\mathrm{g}}(t)}{c_{\mathrm{w}, \text { substrate }}(t) \cdot V_{\mathrm{w}}(t)+c_{\mathrm{g}, \text { substrate }}(t) \cdot V_{\mathrm{g}}(t)+c_{\mathrm{w}, \text { products }}(t) \cdot V_{\mathrm{w}}(t)+c_{\mathrm{g} \text { products }}(t) \cdot V_{\mathrm{g}}(t)} \\
& =\frac{n_{\mathrm{w}, \text { substrate }}(t)+n_{\mathrm{g}, \text { substrate }}(t)}{n_{\mathrm{w}, \text { substrate }}(t)+n_{\mathrm{g}, \text { substrate }}(t)+n_{\mathrm{w}, \text { products }}(t)+n_{\mathrm{g}, \text { products }}(t)}
\end{aligned}
$$

where $n_{\mathrm{g}, \text { substrate }}(t) ; n_{\mathrm{g} \text {,products }}(t)$ and $n_{\mathrm{w}, \text { substrate }}(t) ; n_{\mathrm{w}, \text { products }}(t)$ correspond to the amount of the substrate, degradation products in the gas-phase, and in the water-phase at time $t$, respectively. This correction indirectly accounts for the removal of analytes by sampling, as the substrate amount at time $t$ is not correlated to the initial amount. While this method is exact and circumvents problems arising from imprecisions in sampling volumes and (cumulative) losses of analytes or detector drift during the experiment, its application is limited to systems, where the degradation product(s) are known and can be quantified and the substrate is not used to form biomass.

\section{MATERIALS AND METHODS}

Biodegradation Experiments. Three biodegradation experiments were conducted with pure cultures of Desulfitobacterium hafniense strain Y51, a halorespiring bacterium reducing TCE to cis-DCE. All experiments were set up in $560 \mathrm{~mL}$ serum bottles initially filled with $500 \mathrm{~mL}$ of anoxic medium (see the Supporting Information for details), sealed with butyl stoppers, and stored at $21{ }^{\circ} \mathrm{C}$ in the dark. Each experiment comprised three living replicates and one cell-free control. Experiment 1 was amended with $10 \mathrm{~mL}$ of cell suspension from a non TCE-acclimated preculture grown on organohalide free medium. Experiment 2 was inoculated with $10 \mathrm{~mL}$ of a culture grown in TCE-containing medium. To avoid carry over of organohalides to the batch reactors, the preculture was purged with $\mathrm{N}_{2} / \mathrm{CO}_{2}(80 \% / 20 \%)$ for $1 \mathrm{~h}$ before cell transfer. Experiment 3 was also conducted with TCE-acclimated cells, however, after three consecutive TCE degradation cycles. Each cycle comprised complete TCE degradation, purging of the cell suspension with $\mathrm{N}_{2} / \mathrm{CO}_{2}$ for $1.5 \mathrm{~h}$, and respiking with $20 \mathrm{~mL}$ of an anoxic aqueous TCE solution $(5.6 \mathrm{mM})$. The initial volumetric water-headspace-ratio of Experiment 3 was 0.89 due to sample removal for monitoring of the previous three degradation cycles and readdition of TCE solutions. The conditions of the three experiments are summarized in Table 1, while concentration-plots are provided in Figure SI 1.

Water samples were withdrawn using a gastight glass syringe (Hamilton), and sample aliquots dedicated for concentration analysis were transferred in duplicates to $10 \mathrm{~mL}$ vials (amended with $100 \mu \mathrm{L} \mathrm{H}_{3} \mathrm{PO}_{4}(1 \mathrm{M})$ to stop microbial activity) and sealed using aluminum crimp caps with PTFE-lined septa. Removed water volume was replaced by $\mathrm{N}_{2} / \mathrm{CO}_{2}$ to avoid vacuum. TCE and cis-DCE concentrations were analyzed using a gas chromatograph (TraceGC 2000, Thermo Finnigan, Milano, Italy) coupled to a single-quadrupole mass spectrometer (Trace DSQ, Thermo Finnigan, Austin TX, USA) (GC-MS). The instrument was equipped with a $60 \mathrm{~m} \times 32 \mathrm{~mm}$ RTX-VMS column (Restek Corp., Bellefont, USA). The temperature program was as follows: $5 \mathrm{~min}$ at $40{ }^{\circ} \mathrm{C}, 12{ }^{\circ} \mathrm{C} / \mathrm{min}$ to $150{ }^{\circ} \mathrm{C}$, hold for $2 \mathrm{~min}$. Helium was used as the carrier gas at a flow-rate of $1.5 \mathrm{~mL} / \mathrm{min}$. Applying a static headspace method, $500 \mu \mathrm{L}$ gas phase was injected using a CTC-CombiPAL auto sampler (Chromtech, Idstein, Germany) equipped with a $2.5 \mathrm{~mL}$ headspace syringe. The injector was operated at a constant temperature of $250{ }^{\circ} \mathrm{C}$. Each sample sequence was bracketed by two calibration curves.

One $\mathrm{mL}$ sample aliquots dedicated for carbon isotope analysis were transferred into $1.9 \mathrm{~mL}$ amber vials. To inactivate the strain, the vials were amended with $50 \mu \mathrm{L}$ of $\mathrm{NaOH}(10 \mathrm{M})$. The vials were closed with PTFE-lined screw caps, frozen upside down, and stored at $-20{ }^{\circ} \mathrm{C}$ for subsequent stable carbon isotope analysis. ${ }^{10,26}$ In the GC-IRMS analysis (see the Supporting Information for details) ten to 12 samples were bracketed by external reference standards of TCE $\left(\delta^{13} \mathrm{C}=\right.$ $-26.7 \%$ ) and cis-DCE $\left(\delta^{13} \mathrm{C}=-25.8 \% 0\right)$.

An isotopic mass balance was calculated for the microcosm experiments to validate the consistency of the results

$$
\delta^{13} \mathrm{C}_{\mathrm{TCE}}(0)=\delta^{13} \mathrm{C}_{\mathrm{TCE}}(t) \cdot f_{\mathrm{TCE}}(t)+\delta^{13} \mathrm{C}_{c i s-\mathrm{DCE}}(t) \cdot f_{c i s-\mathrm{DCE}}(t)
$$

where $\delta^{13} \mathrm{C}$ denotes the stable carbon isotope ratio, and $f(t)$ denotes the amount fraction of TCE or cis-DCE. The cis-DCE fraction at each time point $\left(f_{\text {cis-DCE }}(t)\right)$ was calculated using the same correction scheme as for the substrate.

For the stepwise method (method IV) an isotopic mass balance was not calculated as it is not possible to adapt this method in an adequate way to account for the degradation product(s).

Numerical Modeling. First-principle based numerical modeling is an important approach to describe the evolution of concentration and stable isotope ratios both in batch and flow-through systems. ${ }^{4,27-30}$ The importance of modelbased interpretation of biodegradation experiments has been increasingly recognized, and isotope modeling has been performed for a wide range of contaminants such as chlorinated solvents, ${ }^{11,29,31}$ aromatic hydrocarbons, ${ }^{32,33}$ and organic micropollutants. ${ }^{34}$ In this study, we adopt a numerical model to simulate concentration and isotope evolution during microbially mediated TCE dechlorination. The model accounts for substrate degradation and interchange of volatile organic compounds (TCE and cis-DCE) between water- and gasphase, as well as the removal of solute mass and water volume

\begin{tabular}{|c|c|c|c|}
\hline & Expt 1 & Expt 2 & Expt 3 \\
\hline initial amount of TCE $[\mu \mathrm{mol}]^{a}$ & $115 \pm 6$ & $237 \pm 10$ & $103 \pm 3$ \\
\hline $\begin{array}{l}\text { sample volume per time point; } \\
V_{\text {sample }}[\mathrm{mL}]\end{array}$ & 13.7 & 10.1 & 13.7 \\
\hline$\%$ initial phase ratio $\left(V_{\mathrm{g}} / V_{\mathrm{w}}\right)$ & 12 & 12 & 89 \\
\hline $\begin{array}{l}\% \text { (cumulative) amount of initial } \\
\text { TCE sampled }{ }^{b}\end{array}$ & $11.9 \pm 1.4$ & $8.02 \pm 1.3$ & $9.2 \pm 1.4$ \\
\hline $\begin{array}{l}\text { number of sampling points with } \\
\text { TCE; } N\end{array}$ & 15 & 15 & 19 \\
\hline $\begin{array}{l}\% \text { of initial TCE left at last } \\
\text { sampling point }^{c}\end{array}$ & 1.6 & 14.3 & 29.4 \\
\hline
\end{tabular}

Table 1. Conditions and Setups for the Three TCEBiodegradation Experiments Using Desulfitobacterium hafniense Strain Y51

${ }^{a}$ Calculated by $n_{\mathrm{g}}(0)+n_{\mathrm{w}}(0)$ based on the measured data. ${ }^{b}$ Calculated by $n_{\text {rem }}($ total $) /\left(n_{\mathrm{g}}(0)+n_{\mathrm{w}}(0)\right)$. ${ }^{c}$ Calculated by $\left(n_{\mathrm{g}}(t)+n_{\mathrm{w}}(t)\right) /$ $\left(n_{\mathrm{g}}(0)+n_{\mathrm{w}}(0)\right)$. 


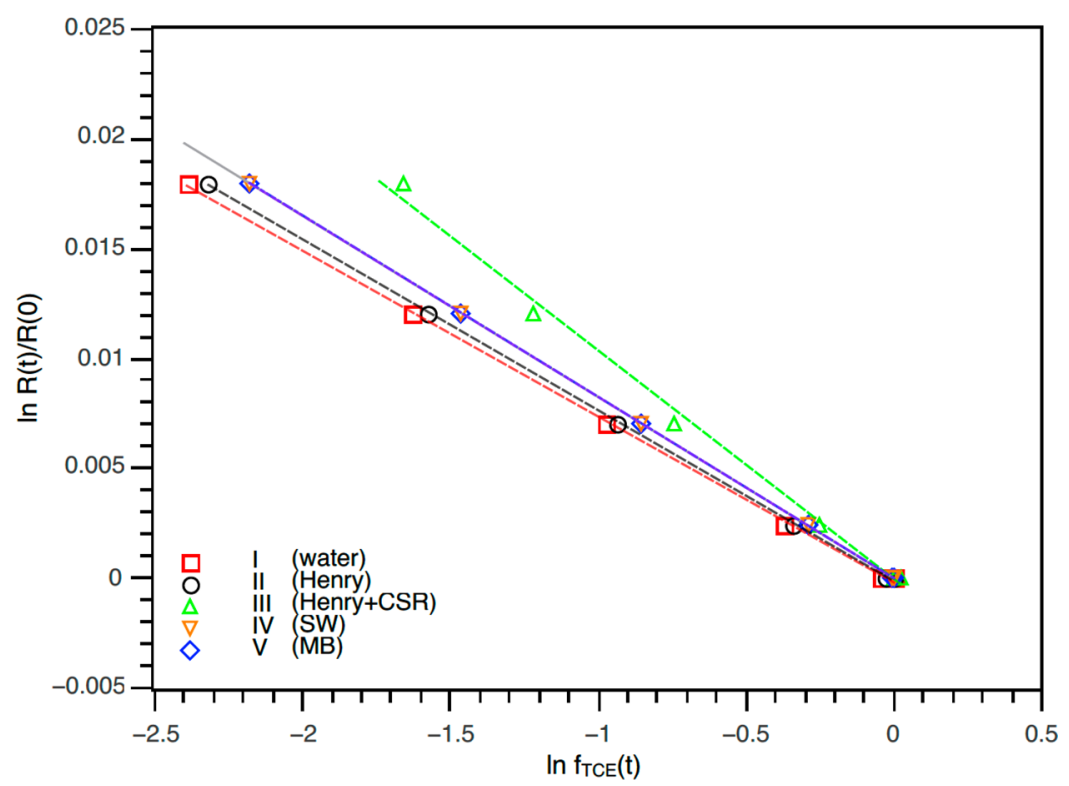

Figure 2. Linearized Rayleigh plot for a data set resulting from a synthetic experiment mimicking biodegradation of TCE to cis-DCE with repetitive sampling of the batch reactor and a "true" isotope enrichment factor of $\varepsilon=-8.25 \%$. The substrate fraction, $f$, was calculated according to ( $\square$ ) amount in water phase (method I, "water"); (O) corrected for gas-water partitioning (method II, "Henry"); $(\triangle)$ corrected for gas-water partitioning and cumulative sample removal (method III, "Henry+CSR"); $(\nabla)$ stepwise method (method IV,"SW"); and $(\diamond)$ mass balance method (method V, "MB"). The solid grey line represents the true $\varepsilon$-value of $-8.25 \%$.

at sampling events. The model was used for the quantitative interpretation of the experimental data and was instrumental in illustrating the need for adequate correction schemes for mass fraction calculations. Furthermore, simulations were used to perform a sensitivity analysis spanning a wide range of experimental parameters of practical importance for improved interpretation and design of isotope studies on degradation of volatile organic compounds. The description of the model formulation and the simulation of the experimental data are reported in the Supporting Information.

\section{RESULTS AND DISCUSSION}

Determination of Isotope Enrichment Factors from a Synthetic Data Set. The effects of substrate fraction correction methods $\mathrm{I}-\mathrm{V}$ on the accuracy in determining the isotope enrichment factor, $\varepsilon$, were evaluated based on a synthetic data set. A known value of $\varepsilon=-8.25 \%$, solely representing isotope fractionation during TCE biodegradation, was used as the key input parameter in the numerical model to generate the synthetic data set. The model mimics real case experimental conditions with interphase mass transfer as well as sample withdrawal and allows producing time wise concentration and isotope data. The model was run for $191 \mathrm{~h}$ in a setup analogous to Experiment 1, with an initial TCE amount of $115 \mu \mathrm{mol}$, an initial aqueous volume of $V_{\mathrm{w}}=500 \mathrm{~mL}$, an initial gas phase volume, $V_{\mathrm{g}}=60 \mathrm{~mL}$, and sample withdrawals $(13.7 \mathrm{~mL}$ each) after $0,48,70,147,165,173,179 \mathrm{~h}$. The output of the model considered in the following analysis are the TCE and cis-DCE aqueous concentrations and carbon isotope ratios at the specified sampling times. This synthetic data set, free of experimental errors, was used to test the performance of the different correction methods to predict the "true" enrichment factor $(\varepsilon=-8.25 \%$ ) . The results are illustrated in Figure 2 as Rayleigh plots.

The different slopes of the linearized Rayleigh equations illustrate that the $\varepsilon$-values varied considerably depending on the
Table 2. Isotope Enrichment Factors $(\varepsilon)$ Resulting from Various Data Evaluation Methods for a Synthetic Biodegradation Experiment Mimicking Reductive Dehalogenation of TCE to cis-DCE with Interphase Mass Transfer, Sample Removal, and a "True" Isotope Enrichment Factor of $\varepsilon=-8.25 \%$ o

\begin{tabular}{lrlc}
\multicolumn{1}{c}{ method } & $\varepsilon[\%$ [ $]$ & CI $(95 \%)$ & $r^{2}$ \\
I (water) & -7.56 & \pm 0.21 & 0.9994 \\
II (Henry) & -7.78 & \pm 0.15 & 0.9997 \\
III (Henry + CSR) & -10.41 & \pm 0.84 & 0.9950 \\
IV (SW) & -8.26 & \pm 0.0001 & 1.0000 \\
V (MB) & -8.26 & \pm 0.003 & 1.0000 \\
\hline
\end{tabular}

correction method applied. Method I (no correction) resulted in $\varepsilon_{\text {water }}=-7.56 \% \pm \pm 0.21 \%$, while a correction for gaswater-partitioning (method II) gave $\varepsilon_{\text {Henry }}=-7.78 \% \pm$ $0.15 \%$. Applying the cumulative sample removal correction (method III) resulted in $\varepsilon_{\text {Henry }+ \text { CSR }}=-10.41 \%$ $\pm 0.84 \%$. The stepwise method (method IV) and the mass balance method (method V) gave similar values: $\varepsilon_{\mathrm{SW}}=-8.26 \% 0 \pm 0.0001 \%$ o and $\varepsilon_{\mathrm{MB}}=-8.26 \% 0 \pm 0.003 \%$ (see Table 2 ).

Thus, the difference between the lowest and highest calculated $\varepsilon$-values was $2.85 \%$ depending on the evaluation method applied for this ideal, error-free synthetic data set. The deviations from the true $\varepsilon$-value $(-8.25 \%)$ used to generate the synthetic data set highlight that methods I and II $\left(\varepsilon_{\text {water; }} \varepsilon_{\text {Henry }}\right)$ underestimate the isotope enrichment factor, whereas method III $\left(\varepsilon_{\text {Henry }+ \text { CSR }}\right)$ overestimates the $\varepsilon$-value. The newly proposed methods IV and V, instead, show an excellent capability to recover the true enrichment factor for TCE degradation. The latter two methods yield enrichment factors of $-8.26 \%$ o that precisely match the true $\varepsilon$-value. These results demonstrate the importance of an accurate calculation of the remaining substrate fraction in batch experiments with repetitive sampling. 

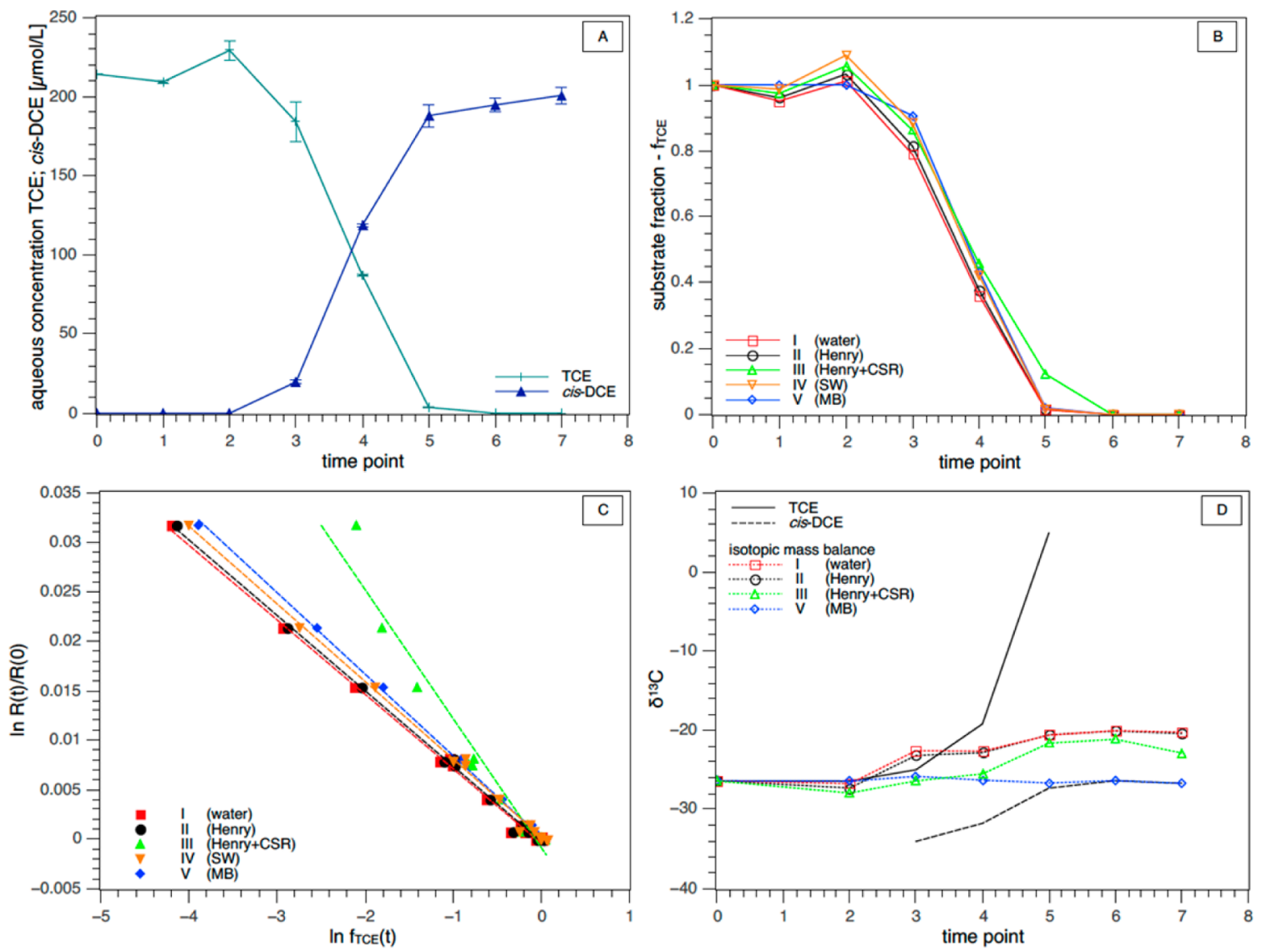

Figure 3. TCE-biodegradation by Desulfitobacterium hafniense Y51 (Experiment 1). (A) Concentration profiles of aqueous TCE (substrate) and cisDCE measured in one (of three) replicate batch assay. Shown are mean values of duplicate GC-MS measurements, while error bars indicate their deviation. (B) Substrate fractions calculated with methods I-V for the data shown in panel A. (C) Rayleigh plots with linear regression lines for methods I-V. All data of the three replicates of Experiment 1 are included. (D) Isotope profile and isotopic mass balance for the data shown in panels $\mathrm{A}$ and $\mathrm{B}$.

Table 3. Compilation of All $\varepsilon$-Values and Their Statistical Parameters Resulting from Data Evaluation Methods I-V for the Three TCE Biodegradation Experiments with Desulfitobacterium hafniense Strain Y51

\begin{tabular}{|c|c|c|c|c|c|c|c|c|c|c|c|c|c|c|c|c|}
\hline \multicolumn{17}{|c|}{ evaluation method } \\
\hline & \multicolumn{3}{|c|}{ I (water) } & \multicolumn{3}{|c|}{ II (Henry) } & \multicolumn{3}{|c|}{ III (Henry + CSR) } & \multicolumn{3}{|c|}{ IV (SW) } & \multicolumn{3}{|c|}{ V (MB) } & \multirow[b]{2}{*}{$\left|\varepsilon_{\max }-\varepsilon_{\min }\right|$} \\
\hline & $\varepsilon[\% o]$ & $\mathrm{CI}$ & $r^{2}$ & $\varepsilon[\% o]$ & $\mathrm{CI}$ & $r^{2}$ & $\varepsilon[\% o]$ & $\mathrm{CI}$ & $r^{2}$ & $\varepsilon[\% o]$ & $\mathrm{CI}$ & $r^{2}$ & $\varepsilon[\% o]$ & $\mathrm{CI}$ & $r^{2}$ & \\
\hline Expt 1 & -7.54 & \pm 0.26 & 0.99 & -7.63 & \pm 0.25 & 0.99 & -13.04 & \pm 1.67 & 0.96 & -7.85 & \pm 0.27 & 0.99 & -8.25 & \pm 0.19 & 0.99 & 5.50 \\
\hline Expt 2 & -7.12 & \pm 0.72 & 0.97 & -7.21 & \pm 0.72 & 0.97 & -9.02 & \pm 1.13 & 0.96 & -7.47 & \pm 0.73 & 0.97 & -8.59 & \pm 0.21 & 0.99 & 1.90 \\
\hline Expt 3 & -5.02 & \pm 1.13 & 0.80 & -6.16 & \pm 1.12 & 0.89 & -8.39 & \pm 1.65 & 0.87 & -5.99 & \pm 1.38 & 0.83 & -8.77 & \pm 0.60 & 0.98 & 3.75 \\
\hline$\left|\varepsilon_{\max }-\varepsilon_{\min }\right|$ & 2.52 & & & 1.47 & & & 4.65 & & & 1.86 & & & 0.52 & & & \\
\hline
\end{tabular}

Experimental Case Studies Illustrating Pitfalls in Determining $\varepsilon$-Values. The critical need of adequate correction schemes to calculate the substrate fraction and the performance of the different methods are demonstrated using experimental data on TCE biodegradation from three different batch experiments. A pure culture of Desulfitobacterium hafniense strain Y51 known for its very robust isotope enrichment factor $(\varepsilon=-8.74 \% \circ \pm 0.32 \% \circ)^{35}$ degraded TCE to cis-DCE.

The time course of measured aqueous concentrations of TCE and cis-DCE in one of the three replicates of Experiment 1 is shown in Figure 3A. The sum of aqueous TCE and cis-DCE concentrations at each time point appears almost constant, which could be misinterpreted as a closed mass balance. However, the cumulative removal of $11.9 \%$ of the initial substrate mass and the effects of volatilization of the compounds into the increasing headspace is masked due to the fact that cis-DCE is 2.6 times less volatile than TCE (see ref 25 and references therein).

Figure 3B illustrates the results of applying the different correction methods to calculate substrate fractions. Significant differences in the time course and the magnitude of the calculated $f$-values were observed for the experimental data shown in Figure 3A. Substrate fractions at early sampling times exceeding unity reveal a weakness of methods that rely on a direct comparison of each measurement in the course of the experiment with the initially measured value. A false measurement of the initial value imposes a systematic error to such methods. 

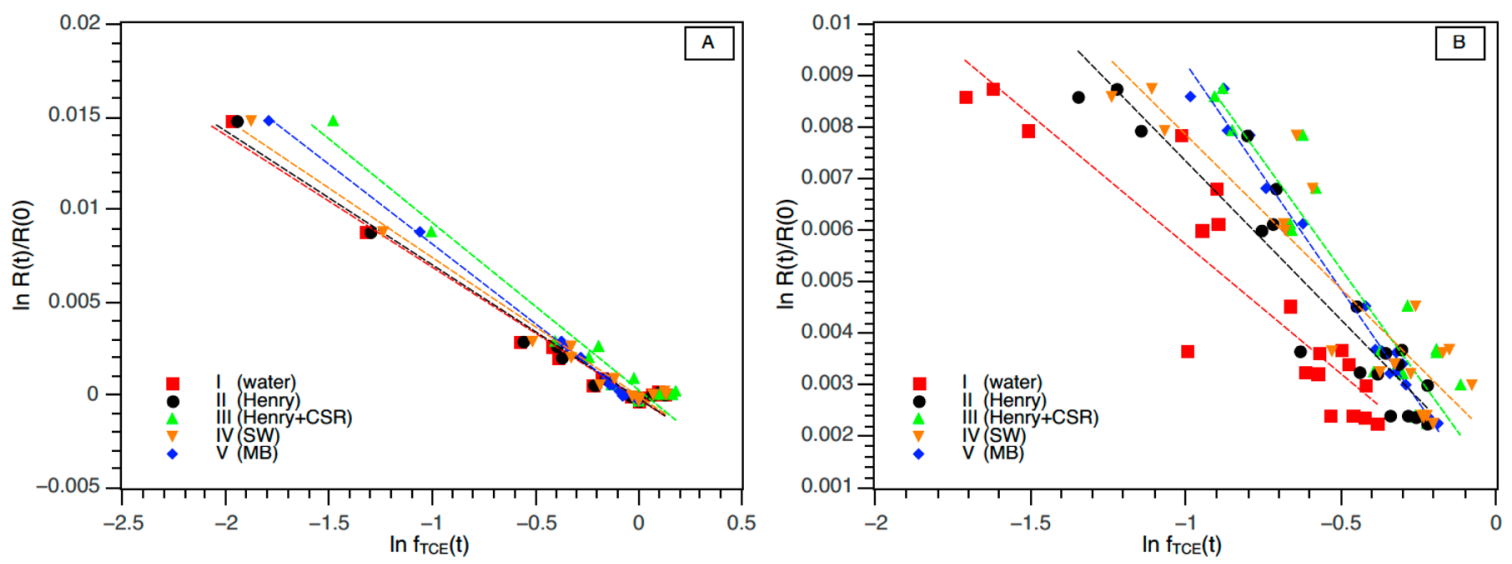

Figure 4. TCE-biodegradation by Desulfitobacterium hafniense strain Y51 (panel A: Experiment 2; panel B: Experiment 3). Rayleigh plots with linear regression lines for methods I-V. All data from three replicates of each experiment are shown $(N=15$ in Expt 2, $N=19$ in Expt 3$)$.

The different evaluation methods for the substrate fraction considerably affect the interpretation of the experimental data based on linear regression in Rayleigh plots (Figure 3C). Methods $\mathrm{I}-\mathrm{V}$, applied considering $f$-values from all three replicates of Experiment 1, show linear trends with different slopes. Thus, distinct $\varepsilon$-values are apparent for the different evaluation methods (see also the results reported in the first row of Table 3 ).

The $\varepsilon$-values derived from the experimental data followed largely the deviations from the true value reported for the synthetic illustrative example discussed above. The smallest $\varepsilon$-value was found for method I (water), while method III (Henry+CSR) gave the highest value. As for the synthetic example, the variability of $\varepsilon$-values was large $(5.5 \%)$ although the statistical parameters $\left(r^{2}>0.96\right.$ and $\left.\mathrm{CI}< \pm 1.67\right)$ for all correction methods indicated low scatter of the data (see Table 3).

Based on statistical reasoning when comparing results of the replicates none of the $\varepsilon$-values can be dismissed as outlier. The $\varepsilon$-values determined for this experimental data set with the evaluation methods IV (SW) and V (MB) were closest to the expected value of $\varepsilon=-8.74 \%$ $\pm 0.32 \%$. However, only method $\mathrm{V}$ matched the expected value with a deviation that can be considered insignificant for experimentally derived $\varepsilon$-values.

As a further criterion of quality control, isotopic mass balances were calculated using different evaluation methods (Figure 3D). A closed isotopic mass balance with an average $\delta^{13} \mathrm{C}$ value of $-26.45 \%$ $\pm 0.12 \%$ o was obtained when the $f$-values of TCE and cis-DCE were calculated using method V. As indicated by shifting $\delta^{13} \mathrm{C}$ values, the isotopic mass balance was not closed using the correction methods I-III. Thus, the isotopic mass balance suggests that the variability of $\varepsilon$-values obtained for the different data evaluation methods is related to a bias in mass balancing of methods I-III for the given data set. Note that method IV does not allow for the calculation of an isotope mass balance which requires quantification of all degradation products.

Two additional biodegradation experiments with pure cultures of Desulfitobacterium hafniense strain Y51 were conducted to further assess the performance of data evaluation methods I-V on experimental data sets (for experimental conditions see Table 1). The data are reported as Rayleigh plots in Figure 4, for Experiment 2 (panel A) and for Experiment 3 (panel B). Further details and graphical representations of the results of these experiments are reported in the Supporting Information
(Figures SI2 and SI3). The overall variation of the isotope enrichment factors obtained was smaller compared to Experiment 1 (see right column Table 3), but similar trends as discussed for Experiment 1 were consistently found also for Experiment 2 and Experiment 3.

Again, methods I and II resulted in lowest isotope fractionation (small absolute $\varepsilon$-values), while method $\mathrm{V}$ was closest to the expected $\varepsilon$-value. A comparison of the variability of a given evaluation method for the three different experiments (see bottom line Table 3) indicates that method $\mathrm{V}$ showed both the lowest variability and the smallest deviation from the expected value, whereas method III varied most and thus provided the least consistent estimates of $\varepsilon$-values. Methods I, II, and IV showed similar variability but different degrees of mismatch with respect to the expected value. Even though method IV (SW) was proven to be correct for an error free synthetic data set, this method failed to reproduce the expected value in the experimental case studies. This mismatch is related to the iterative correction scheme of method IV requiring a high precision of the withdrawn volumes as well as of the measured substrate concentrations. Method V (MB) circumvents these experimental error sources, but its application is limited to systems, where the degradation product(s) are known and can be quantified.

Thus, the evaluation of the data from Experiments 1-3 resulted in a considerable variability of the estimated $\varepsilon$-values, even when carried out with the same evaluation method. For most evaluation methods, this difference is striking since the experiments were carried out under similar conditions using the same microbial strain. This outcome points to a high sensitivity of the data evaluation procedure toward specific values of experimental parameters such as the total amount of substrate removed by sampling, sampling volume, number of sampling points, or the amount of initial TCE left at the last sampling point, which were not the same in the three experimental setups.

Sensitivity Analysis and Environmental Implications. The evaluation of the synthetic data set and the experimental case studies revealed a significant impact of the data evaluation method on both the magnitude and the consistency of the obtained isotope enrichment factors. The case studies emphasized that the degree of variation of the enrichment factor depends on the experimental conditions and on the quality and consistency of the experimental data. Thus, we performed a comprehensive model-based sensitivity analysis to evaluate the 
Table 4. Sensitivity Analysis of Experimental Parameters on Isotope Enrichment Factors Calculated Using Different Data Evaluation Methods ${ }^{a}$

\begin{tabular}{|c|c|c|c|c|c|c|c|c|c|c|c|c|c|c|c|c|c|c|c|c|c|c|c|c|c|}
\hline \multirow{2}{*}{$\begin{array}{c}\text { Parameter } \\
\text { varied } \\
\text { Evaluation } \\
\text { method }\end{array}$} & \multicolumn{5}{|c|}{$\begin{array}{l}\text { Initial phase ratio } \\
\qquad\left(V_{g} / V_{w}\right)\end{array}$} & \multicolumn{5}{|c|}{$\begin{array}{l}\text { Volume fraction (in \%) of an } \\
\text { individual sample compared to the } \\
\text { initial water volume of the reactor } \\
\left(V_{\text {sample }} / V_{w} \text {; initial }\right)\end{array}$} & \multicolumn{5}{|c|}{$\begin{array}{l}\text { Number of sampling points } \\
\text { (N) }\end{array}$} & \multicolumn{5}{|c|}{$\begin{array}{l}\% \text { of initial TCE left at last sampling } \\
\text { point }(n(t) / n(0))\end{array}$} & \multicolumn{5}{|c|}{$\begin{array}{c}\text { Henry constant } \\
\text { (dimensionless; } c_{w} / c_{g} \text { ) }\end{array}$} \\
\hline & $\begin{array}{c}\text { I } \\
\text { water }\end{array}$ & $\begin{array}{c}\text { II } \\
\text { Henry }\end{array}$ & $\begin{array}{c}\text { III } \\
\text { CSR }\end{array}$ & $\begin{array}{l}\text { IV } \\
\text { SW }\end{array}$ & $\begin{array}{c}\mathrm{V} \\
\mathrm{MB}\end{array}$ & $\begin{array}{c}\text { I } \\
\text { water }\end{array}$ & $\begin{array}{c}\text { II } \\
\text { Henry }\end{array}$ & $\begin{array}{c}\text { III } \\
\text { CSR }\end{array}$ & $\begin{array}{l}\text { IV } \\
\text { SW }\end{array}$ & $\begin{array}{c}\mathrm{V} \\
\mathrm{MB}\end{array}$ & $\begin{array}{c}\text { I } \\
\text { water }\end{array}$ & $\begin{array}{c}\text { II } \\
\text { Henry }\end{array}$ & $\begin{array}{c}\text { III } \\
\text { CSR }\end{array}$ & $\begin{array}{l}\text { IV } \\
\text { SW }\end{array}$ & $\begin{array}{c}\mathrm{v} \\
\mathrm{MB}\end{array}$ & $\begin{array}{c}\mathrm{I} \\
\text { water }\end{array}$ & $\begin{array}{c}\text { II } \\
\text { Henry }\end{array}$ & $\begin{array}{l}\text { III } \\
\text { CSR }\end{array}$ & $\begin{array}{l}\text { IV } \\
\text { SW }\end{array}$ & $\begin{array}{c}\mathrm{V} \\
\mathrm{MB}\end{array}$ & $\begin{array}{c}\mathrm{I} \\
\text { water }\end{array}$ & $\begin{array}{c}\text { II } \\
\text { Henry }\end{array}$ & $\begin{array}{c}\text { III } \\
\text { CSR }\end{array}$ & $\begin{array}{l}\text { IV } \\
\text { SW }\end{array}$ & $\begin{array}{c}\mathrm{V} \\
\mathrm{MB}\end{array}$ \\
\hline \multirow[b]{2}{*}{ Scenario 1} & \multicolumn{5}{|c|}{$V_{g} / V_{w}=0.05$} & \multicolumn{5}{|c|}{$V_{\text {sample }} / V_{w}$; initial $=0.4 \%$} & \multicolumn{5}{|c|}{$\mathrm{N}=3$} & \multicolumn{5}{|c|}{$44 \%$} & \multicolumn{5}{|c|}{87.41 (hexachlorobenzene) } \\
\hline & -7.58 & -7.79 & -10.57 & -8.26 & -8.26 & -8.16 & -8.19 & -8.62 & -8.26 & -8.26 & -7.91 & -8.06 & -9.39 & -8.26 & -8.26 & -6.39 & -6.94 & -9.38 & -8.26 & -8.27 & -7.82 & -7.82 & -11.06 & -8.26 & -8.25 \\
\hline \multirow{2}{*}{ Base case } & \multicolumn{5}{|c|}{$V_{g} / V_{w}=0.12$} & \multicolumn{5}{|c|}{$V_{\text {sample }} / V_{w} ;$ initial $=2.74 \%$} & \multicolumn{5}{|c|}{$\mathrm{N}=7$} & \multicolumn{5}{|c|}{$10 \%$} & \multicolumn{5}{|c|}{2.34 (TCE) } \\
\hline & -7.56 & -7.78 & -10.41 & -8.26 & -8.26 & -7.56 & -7.78 & -10.41 & -8.26 & -8.26 & -7.56 & -7.78 & -10.41 & -8.26 & -8.26 & -7.56 & -7.78 & -10.41 & -8.26 & -8.26 & -7.56 & -7.78 & -10.41 & -8.26 & -8.26 \\
\hline \multirow{2}{*}{ Scenario 2} & \multicolumn{5}{|c|}{$V_{g} / V_{w}=0.3$} & \multicolumn{5}{|c|}{$V_{\text {sample }} V_{w}$; initial $=4 \%$} & \multicolumn{5}{|c|}{$\mathrm{N}=15$} & \multicolumn{5}{|c|}{$1 \%$} & \multicolumn{5}{|c|}{1.12 (vinyl chloride) } \\
\hline & -7.50 & -7.77 & -9.75 & -8.26 & -8.27 & -7.18 & -7.53 & -11.22 & -8.26 & -8.28 & -6.10 & -6.78 & -12.58 & -8.26 & -8.32 & -7.91 & -8.03 & -13.45 & -8.26 & -8.26 & -7.26 & -7.75 & -9.95 & -8.26 & -8.28 \\
\hline$\left(\varepsilon-\varepsilon_{\text {true }}\right)_{\max }$ & 0.8 & 0.5 & -2.3 & -0.01 & -0.02 & 1.1 & 0.7 & -3.0 & -0.01 & -0.03 & 2.4 & 1.5 & -4.3 & -0.01 & -0.07 & 2.8 & 2.2 & -5.2 & 0.01 & 0.02 & 1.0 & 0.5 & -2.8 & -0.01 & -0.02 \\
\hline
\end{tabular}

${ }^{a_{T}}$ The base case refers to the parameter values of the synthetic experiment shown in Figure 1 and Table 2. The parameter values considered in scenarios 1 and 2 bracket the bases case within a realistic band with. Only one parameter is varied while keeping all others at the values of the base case. The "true" isotope enrichment factor used to calculate the synthetic data sets $\left(\varepsilon_{\text {true }}\right)$ is $-8.25 \%$.

impact of different experimental conditions and to derive guidelines for proper experimental design and data evaluation. We used the described numerical model to simulate biodegradation of volatile compounds in batch systems with the aim of systematically assessing the influence of key selected parameters on the interpretation of isotope enrichment factors. The following experimental parameters were considered: initial phase ratio $\left(V_{\mathrm{g}} / V_{\mathrm{w}}\right)$, relative sample volume withdrawn per time point $\left(V_{\text {sample }} / V_{\mathrm{w} \text {,initial }}\right)$, number of sampling points, percentage of initial substrate left at the last sampling point $(n(t) / n(0))$, and the tendency of the analyte to partition between the water and the gas phase (dimensionless Henry constant).

TCE biodegradation in the same setup and with the same parameters as in the synthetic experiment was considered as base case scenario for the sensitivity analysis. In the sensitivity analysis each parameter was varied within a meaningful range in two distinct scenarios around the base case, while leaving all other parameters unchanged. For each of the resulting data sets $\varepsilon$-values were calculated applying the correction methods $\mathrm{I}-\mathrm{V}$. The results of the sensitivity analysis are summarized in Table 4, while a graphical representation is provided in the SI.

For all constellations considered, the evaluation methods I-III deviated significantly from the true $\varepsilon$-value $(-8.25 \%$ o). While methods I and II always underestimated the true $\varepsilon$, method III strongly overestimated the enrichment factor. The over- or underestimation of substrate fraction is conceptually inherent to methods I-III as the removed mass is not accurately considered. While methods I and II treat the removed substrate as biodegraded, method III assumes that it is still present in the system. Table 4 further shows that applying an inappropriate data evaluation method not only leads to inaccurate (i.e., large deviation from the true $\varepsilon$-value) but also to inconsistent results (i.e., with a given method different $\varepsilon$-values are obtained for different experimental parameters). To illustrate the latter effect, we take a closer look at the impact of the percentage of initial TCE left at the last sampling point. Well-designed (bio)-degradation batch experiments comprise a number of replicates in order to evaluate reproducibility and to calculate mean values and standard deviations of the results. The progress of the transformation reaction, however, may vary considerably among the replicates leading to different degrees of (bio)-degradation at the last sampling point. The data presented in Table 4 clearly demonstrate that such conditions lead to very different $\varepsilon$-values if one of the methods I-III is used for data evaluation. Such experiments are prone to misinterpretations, e.g., that $\varepsilon$-values depend on degradation rates or that different enzymatic systems are involved at different degradation rates. Thus, the choice of an unsuitable data evaluation method may lead to artifacts even when strictly defined experimental protocols are applied. This pitfall is avoided when using appropriate correction schemes such as methods IV and V for which the estimated $\varepsilon$-values did not change at different extents of progress of the transformation reaction.

Overall, the sensitivity analysis demonstrates that only methods IV (SW) and V (MB) are suitable to accurately determine the isotope enrichment factor of volatile compounds. The maximum variation of the estimated $\varepsilon$-values from the true (see last row of Table 4) clearly indicates that these two proposed methods outperform the other methods (I-III) and provide accurate and consistent estimates of the enrichment factor over a wide range of tested scenarios and conditions.

Based on the experimental and modeling results obtained in this study, we suggest the following recommendations for (bio)-degradation experiments of volatile organic compounds: (i) Generally, batch experiments with repetitive sample removal should be designed to minimize the ratio of removed to initial amount of the substrate; (ii) either method IV (SW) or method V (MB) should be applied to evaluate isotope data in batch experiments with volatile organic compounds based on a Rayleigh equation; (iii) method V (MB) should be adopted if all degradation products can be quantified. This method is robust against errors related to sample removal as well as analytical errors in concentration measurements, e.g., due to detector drifts. Furthermore, method V allows verifying the quality of the data by an isotopic mass balance; (iv) method IV (SW) should be selected for experiments where only substrate concentrations can be monitored. This method, however, requires highly accurate data of the withdrawn volumes and of the analyzed substrate concentrations.

The results presented here demonstrate that even with wellcontrolled laboratory batch experiments and with highly precise and reproducible concentration and isotope measurements the determination of isotope enrichment factors is prone to significant systematic errors. Choosing an inappropriate method to evaluate measured concentration data may not only lead to inconsistent but also to incorrect $\varepsilon$-values and thus to misinterpretations regarding the processes underlying isotope fractionation. Regarding the variability of published isotope enrichment factors for a given microcosm system, pure culture, or abiotic transformation process our results suggest that a 
re-evaluation of raw data from experiments with repetitive sample removal might reveal more consistent $\varepsilon$-values due to the elimination of potential artifacts arising from inappropriate data evaluation.

\section{ASSOCIATED CONTENT}

\section{S Supporting Information}

The Supporting Information is available free of charge on the ACS Publications website at DOI: 10.1021/acs.est.6b03689.

Additional information on cultivation conditions of D. hafniense strain Y51, analytical methods (GC-MS and $\delta^{13} \mathrm{C}$ isotope analyses), additional results on the biodegradation experiments, additional information on the model formulation and data interpretation, a graphical representation of the sensitivity analysis as well as an evaluation of propagated statistical uncertainty (error propagation) (PDF)

\section{AUTHOR INFORMATION}

\section{Corresponding Author}

*Phone +49-7071-2973148. E-mail: haderlein@uni-tuebingen. de. Corresponding author address: Department of Geosciences Center for Applied Geosciences, University of Tübingen, Hölderlinstrasse 12, D-72070 Tübingen, Germany.

\section{ORCID ${ }^{\circ}$}

Stefan B. Haderlein: 0000-0002-9309-8523

\section{Present Addresses}

${ }^{\S}$ Karin Ebert, BoSS Consult GmbH, Lotterbergstraße 16, D-70499 Stuttgart, Germany.

\section{Notes}

The authors declare no competing financial interest.

\section{ACKNOWLEDGMENTS}

We thank Dr. Christine Laskov for helpful discussions and comments on an earlier version of the manuscript. Financial support was provided by the German Research Foundation (Grants HA3453/12-1, BE 4350/3-1; INST 37/740-1 FUGG; RO 4169/2-1) and by the State of Baden-Württemberg (LGFG grants to D.B. and K.E). B.J. and M.R. acknowledge funding by the Department of Environmental Engineering at the Technical University of Denmark. Prof. Furukawa, Department of Bioscience and Biotechnology Kyushu University (Japan), kindly provided the strain Desulfitobacterium hafniense Y51.

\section{REFERENCES}

(1) Schmidt, T. C.; Zwank, L.; Elsner, M.; Berg, M.; Meckenstock, R. U.; Haderlein, S. B. Compound-specific stable isotope analysis of organic contaminants in natural environments: a critical review of the state of the art, prospects, and future challenges. Anal. Bioanal. Chem. 2004, 378 (2), 283-300.

(2) Blessing, M.; Schmidt, T. C.; Dinkel, R.; Haderlein, S. B. Delineation of Multiple Chlorinated Ethene Sources in an Industrialized Area- A Forensic Field Study Using Compound-Specific Isotope Analysis. Environ. Sci. Technol. 2009, 43 (8), 2701-2707.

(3) McKelvie, J. R.; Mackay, D. M.; de Sieyes, N. R.; LacrampeCouloume, G.; Sherwood Lollar, B. Quantifying MTBE biodegradation in the Vandenberg Air Force Base ethanol release study using stable carbon isotopes. J. Contam. Hydrol. 2007, 94 (3-4), 157-165.

(4) Thullner, M.; Centler, F.; Richnow, H.-H.; Fischer, A. Quantification of organic pollutant degradation in contaminated aquifers using compound-specific stable isotope analysis - Review of recent developments. Org. Geochem. 2012, 42 (12), 1440-1460.
(5) Richnow, H. H.; Annweiler, E.; Michaelis, W.; Meckenstock, R. U. Microbial in situ degradation of aromatic hydrocarbons in a contaminated aquifer monitored by carbon isotope fractionation. $J$. Contam. Hydrol. 2003, 65 (1-2), 101-120.

(6) Maillard, J.; Schumacher, W.; Vazquez, F.; Regeard, C.; Hagen, W. R.; Holliger, C. Characterization of the corrinoid iron-sulfur protein tetrachloroethene reductive dehalogenase of Dehalobacter restrictus. Appl. Environ. Microbiol. 2003, 69 (8), 4628.

(7) Nijenhuis, I.; Andert, J.; Beck, K.; Kastner, M.; Diekert, G.; Richnow, H. H. Stable isotope fractionation of tetrachloroethene during reductive dechlorination by Sulfurospirillum multivorans and Desulfitobacterium sp. strain PCE-S and abiotic reactions with cyanocobalamin. Appl. Environ. Microbiol. 2005, 71 (7), 3413.

(8) Cichocka, D.; Siegert, M.; Imfeld, G.; Andert, J.; Beck, K.; Diekert, G.; Richnow, H. H.; Nijenhuis, I. Factors controlling the carbon isotope fractionation of tetra-and trichloroethene during reductive dechlorination by Sulfurospirillum ssp. Desulfitobacterium sp. strain PCE-S. FEMS Microbiol. Ecol. 2007, 62 (1), 98-107.

(9) Kampara, M.; Thullner, M.; Harms, H.; Wick, L. Y. Impact of cell density on microbially induced stable isotope fractionation. Appl. Microbiol. Biotechnol. 2009, 81 (5), 977-985.

(10) Cretnik, S.; Thoreson, K. A.; Bernstein, A.; Ebert, K.; Buchner, D.; Laskov, C.; Haderlein, S.; Shouakar-Stash, O.; Kliegman, S.; McNeill, K.; et al. Reductive Dechlorination of TCE by Chemical Model Systems in Comparison to Dehalogenating Bacteria: Insights from Dual Element Isotope Analysis (13C/12C, 37Cl/35Cl). Environ. Sci. Technol. 2013, 47 (13), 6855-6863.

(11) Kuder, T.; van Breukelen, B. M.; Vanderford, M.; Philp, P. 3DCSIA: carbon, chlorine, and hydrogen isotope fractionation in transformation of TCE to ethene by a Dehalococcoides culture. Environ. Sci. Technol. 2013, 47 (17), 9668-9677.

(12) Lee, P. K. H.; Conrad, M. E.; Alvarez-Cohen, L. Stable carbon isotope fractionation of chloroethenes by dehalorespiring isolates. Environ. Sci. Technol. 2007, 41 (12), 4277-4285.

(13) Harding, K. C.; Lee, P. K. H.; Bill, M.; Buscheck, T. E.; Conrad, M. E.; Alvarez-Cohen, L. Effects of Varying Growth Conditions on Stable Carbon Isotope Fractionation of Trichloroethene (TCE) by tce A-containing Dehalococcoides mccartyi strains. Environ. Sci. Technol. 2013, 47 (21), 12342-12350.

(14) Fletcher, K. E.; Nijenhuis, I.; Richnow, H. H.; Loeffler, F. E. Stable Carbon Isotope Enrichment Factors for cis-1, 2-Dichloroethene and Vinyl Chloride Reductive Dechlorination by Dehalococcoides. Environ. Sci. Technol. 2011, 45 (7), 2951-2957.

(15) Sherwood Lollar, B.; Hirschorn, S.; Mundle, S. O. C.; Grostern, A.; Edwards, E. A.; Lacrampe-Couloume, G. Insights into Enzyme Kinetics of Chloroethane Biodegradation Using Compound Specific Stable Isotopes. Environ. Sci. Technol. 2010, 44 (19), 7498-7503.

(16) Thullner, M.; Kampara, M.; Richnow, H. H.; Harms, H.; Wick, L. Y. Impact of Bioavailability Restrictions on Microbially Induced Stable Isotope Fractionation. 1. Theoretical Calculation. Environ. Sci. Technol. 2008, 42 (17), 6544-6551.

(17) Mancini, S. A.; Hirschorn, S. K.; Elsner, M.; LacrampeCouloume, G.; Sleep, B. E.; Edwards, E. A.; Sherwood Lollar, B. Effects of trace element concentration on enzyme controlled stable isotope fractionation during aerobic biodegradation of toluene. Environ. Sci. Technol. 2006, 40 (24), 7675-7681.

(18) Rayleigh, L. Theoretical considerations respecting the separation of gases by diffusion and similar processes. London, Edinburgh, Dublin Philos. Mag. J. Sci. 1896, 42 (259), 493-498.

(19) Mariotti, A.; Germon, J. C.; Hubert, P.; Kaiser, P.; Letolle, R.; Tardieux, A.; Tardieux, P. Experimental determination of nitrogen kinetic isotope fractionation: some principles; illustration for the denitrification and nitrification processes. Plant Soil 1981, 62 (3), 413-430.

(20) Van Breukelen, B. M. Quantifying the degradation and dilution contribution to natural attenuation of contaminants by means of an open system Rayleigh equation. Environ. Sci. Technol. 2007, 41 (14), $4980-4985$ 
(21) Bloom, Y.; Aravena, R.; Hunkeler, D.; Edwards, E.; Frape, S. K. Carbon isotope fractionation during microbial dechlorination of trichloroethene, cis-1, 2-dichloroethene, and vinyl chloride: implications for assessment of natural attenuation. Environ. Sci. Technol. 2000, 34 (13), 2768-2772.

(22) Chu, K.-H.; Mahendra, S.; Song, D. L.; Conrad, M. E.; AlvarezCohen, L. Stable Carbon Isotope Fractionation during Aerobic Biodegradation of Chlorinated Ethenes. Environ. Sci. Technol. 2004, 38 (11), 3126-3130.

(23) Mundle, S. O. C.; Johnson, T.; Lacrampe-Couloume, G.; Pérezde-Mora, A.; Duhamel, M.; Edwards, E. A.; McMaster, M. L.; Cox, E.; Révész, K.; Sherwood Lollar, B. Monitoring biodegradation of ethene and bioremediation of chlorinated ethenes at a contaminated site using compound-specific isotope analysis (CSIA). Environ. Sci. Technol. 2012, 46 (3), 1731-1738.

(24) Scott, K. M.; Lu, X.; Cavanaugh, C. M.; Liu, J. S. Optimal methods for estimating kinetic isotope effects from different forms of the Rayleigh distillation equation. Geochim. Cosmochim. Acta 2004, 68 (3), 433-442.

(25) Sander, R. Compilation of Henry's law constants (version 4.0) for water as solvent. Atmos. Chem. Phys. 2015, 15 (8), 4399-4981.

(26) Elsner, M.; Couloume, G. L.; Sherwood Lollar, B. Freezing to preserve groundwater samples and improve headspace quantification limits of water-soluble organic contaminants for carbon isotope analysis. Anal. Chem. 2006, 78 (21), 7528-7534.

(27) Van Breukelen, B. M.; Hunkeler, D.; Volkering, F. Quantification of Sequential Chlorinated Ethene Degradation by Use of a Reactive Transport Model Incorporating Isotope Fractionation. Environ. Sci. Technol. 2005, 39 (11), 4189-4197.

(28) Van Breukelen, B. M.; Rolle, M. Transverse hydrodynamic dispersion effects on isotope signals in groundwater chlorinated solvents' plumes. Environ. Sci. Technol. 2012, 46 (14), 7700-7708.

(29) Aeppli, C.; Berg, M.; Cirpka, O. A.; Holliger, C.; Schwarzenbach, R. P.; Hofstetter, T. B. Influence of mass-transfer limitations on carbon isotope fractionation during microbial dechlorination of trichloroethene. Environ. Sci. Technol. 2009, 43 (23), 8813-8820.

(30) Eckert, D.; Rolle, M.; Cirpka, O. A. Numerical simulation of isotope fractionation in steady-state bioreactive transport controlled by transverse mixing. J. Contam. Hydrol. 2012, 140-141, 95-106.

(31) Jin, B.; Haderlein, S. B.; Rolle, M. Integrated carbon and chlorine isotope modeling: applications to chlorinated aliphatic hydrocarbons dechlorination. Environ. Sci. Technol. 2013, 47 (3), $1443-1451$.

(32) Jin, B.; Rolle, M.; Li, T.; Haderlein, S. B. Diffusive fractionation of BTEX and chlorinated ethenes in aqueous solution: quantification of spatial isotope gradients. Environ. Sci. Technol. 2014, 48 (11), 61416150.

(33) Mellage, A.; Eckert, D.; Grösbacher, M.; Inan, A. Z.; Cirpka, O. A.; Griebler, C. Dynamics of suspended and attached aerobic toluene degraders in small-scale flow-through sediment systems under growth and starvation conditions. Environ. Sci. Technol. 2015, 49 (12), 71617169.

(34) Jin, B.; Rolle, M. Position-specific isotope modeling of organic micropollutants transformation through different reaction pathways. Environ. Pollut. 2016, 210, 94-103.

(35) Buchner, D.; Behrens, S.; Laskov, C.; Haderlein, S. B. Resiliency of Stable Isotope Fractionation $(\delta(13) \mathrm{C}$ and $\delta(37) \mathrm{Cl})$ of Trichloroethene to Bacterial Growth Physiology and Expression of Key Enzymes. Environ. Sci. Technol. 2015, 49 (22), 13230-13237. 